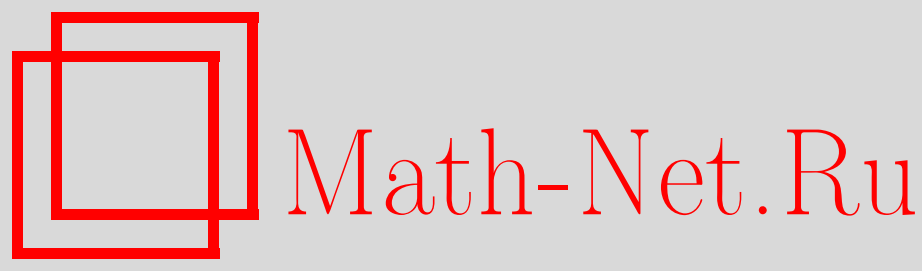

У. А. Розиков, Г. Т. Мадгозиев, Неединственность меры Гиббса для одной модели на дереве Кэли, ТМФ, 2011, том 167, номер 2, 311-322

DOI: https://doi.org/10.4213/tmf6642

Использование Общероссийского математического портала Math-Net.Ru подразумевает, что вы прочитали и согласны с пользовательским соглашением http://www.mathnet.ru/rus/agreement

Параметры загрузки:

IP : 35.174 .16 .151

26 апреля 2023 г., 16:38:56

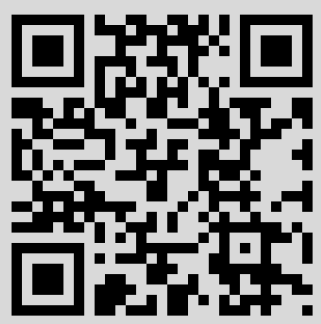


Том 167, № 2

май, 2011

(C) 2011 г.

У. А. Розиков*, Г. Т. Мадгозиев*

\title{
НЕЕДИНСТВЕННОСТЬ МЕРЫ ГИББСА ДЛЯ ОДНОЙ МОДЕЛИ НА ДЕРЕВЕ КЭЛИ
}

\begin{abstract}
Предложена модель на дереве Кэли и показано, что для нее существует несчетное множество $\widehat{G}$-периодических гиббсовских мер в отличие от ранее изученных моделей.
\end{abstract}

Ключевые слова: дерево Кэли, конфигурация, НС-модель, мера Гиббса.

\section{1. ВВЕДЕНИЕ}

Дерево Кэли $\Gamma^{k}=(V, L)$ порядка $k \geqslant 1$ - бесконечное дерево, т.е. граф без циклов, из каждой вершины которого выходит ровно $k+1$ ребро. Здесь $V$ есть множество вершин $\Gamma^{k}, L$ - множество его ребер. Пусть $i$ - функция инцидентности, сопоставляющая каждому ребру $l \in L$ его концевые точки $x, y \in V$. Если $i(l)=\{x, y\}$, то вершины $x$ и $y$ называются ближайшими соседями и обозначаются через $\langle x, y\rangle$. Расстояние $d(x, y), x, y \in V$, на дереве Кэли определяется формулой

$$
d(x, y)=\min \left\{d \mid \exists x=x_{0}, x_{1}, \ldots, x_{d-1}, x_{d}=y \in V \text { так, что }\left\langle x_{0}, x_{1}\right\rangle, \ldots,\left\langle x_{d-1}, x_{d}\right\rangle\right\} .
$$

Известно, что дерево Кэли представляется как группа $G_{k}$, являющаяся свободным произведением $k+1$ циклической группы второго порядка с образующими $a_{1}, a_{2}, \ldots, a_{k+1}[1]$.

Пусть $\Phi-$ конечное множество. Конфигурация $\sigma$ на $V$ определяется как функция $x \in V \rightarrow \sigma(x) \in \Phi$. Множество всех конфигураций совпадает с $\Omega=\Phi^{V}$. Пусть $A \subset V$. Обозначим через $\Omega_{A}$ пространство конфигураций, определенных на множестве $A$. Через $|A|$ обозначим число элементов множества $A$.

Определим обобщенный символ Кронекера как функцию

$$
U\left(\sigma_{A}\right): \Omega_{A} \rightarrow\{|A|-1,|A|-2, \ldots,|A|-\min \{|A|,|\Phi|\}\}
$$

следующим образом:

$$
U\left(\sigma_{A}\right)=|A|-\left|\sigma_{A} \cap \Phi\right|,
$$

где $A \subset V$ и $\left|\sigma_{A} \cap \Phi\right|$ - число различных значений $\sigma_{A}(x), x \in A[2]$.

* Институт математики и информационных технологий АНРУз, Ташкент, Узбекистан. E-mail: gmadgoziyev@yandex.ru 
В настоящей работе мы рассматриваем случай $\Phi=\{-1,1\}$ и $|A|=4$. Обозначим через $M$ множество всех шаров $b(x)=\{y \in V: d(x, y) \leqslant 1\}$ с радиусом 1 .

Гамильтониан определяется следующим образом:

$$
H(\sigma)=-J \sum_{b \in M} U\left(\sigma_{b}\right)
$$

где $J>0$.

Основной проблемой данного гамильтониана является описание всех отвечающих ему мер Гиббса. Определение меры Гиббса и других понятий, связанных с теорией мер Гиббса, можно найти, например, в работе [3]. Для модели Изинга на дереве Кэли эта задача изучена достаточно полно. Так, например, в работе [4] построено несчетное множество крайних гиббсовских мер, в работе [5] найдено необходимое и достаточное условие крайности неупорядоченной фазы модели Изинга на дереве Кэли.

Цель настоящей работы - изучение меры Гиббса для модели (1.2). Эта модель контурным методом изучена в работе [2], здесь мы преобразуем ее в HC- (hard-core) модель на дереве Кэли и применим метод марковских случайных полей и рекуррентных уравнений этой теории (см., например, работы [1], [3]-[9]).

\section{2. СИСТЕМА ФУНКЦИОНАЛЬНЫХ УРАВНЕНИЙ}

Пусть $x^{0} \in V$ - фиксированная точка. Будем писать $x<y$, если путь от $x^{0}$ до $y$ проходит через $x$. Вершина $y$ называется "прямым потомком" $x$, если $y>x$ и $x, y$ являются соседями.

Обозначим

$$
W_{n}=\left\{x \in V: d\left(x^{0}, x\right)=n\right\}, \quad V_{n}=\left\{x \in V: d\left(x^{0}, x\right) \leqslant n\right\} .
$$

Для $x \in G_{k}$ положим $x_{\downarrow}=\left\{y \in G_{k}:\langle x, y\rangle\right\} \backslash S(x)$, где $S(x)$ - множество прямых потомков точки $x \in V$.

В этой работе мы рассмотрим случай $k=2$. Пусть $b(x)=\left\{x, x a_{1}, x a_{2}, x a_{3}\right\}$ и $\sigma_{b(x)}=\left\{\sigma(x), \sigma\left(a x_{1}\right), \sigma\left(a x_{2}\right), \sigma\left(a x_{3}\right)\right\}$.

Рассмотрим вероятностное распределение $\mu^{(n)}$ на $\Omega_{V_{n}}$ :

$$
\mu^{(n)}\left(\sigma_{n}\right)=Z_{n}^{-1} \exp \left\{-\beta H\left(\sigma_{n}\right)+\sum_{x \in W_{n}} h_{b(x), \sigma_{b(x)}}^{\sigma_{b(x \downarrow)}}\right\},
$$

где $\sigma_{n} \in \Omega_{V_{n}}$,

$$
Z_{n}=\sum_{\bar{\sigma}_{n} \in \Omega_{V_{n}}} \exp \left\{-\beta H\left(\bar{\sigma}_{n}\right)+\sum_{x \in W_{n}} h_{b(x), \bar{\sigma}_{b(x)}}^{\bar{\sigma}_{b\left(x_{\downarrow}\right)}}\right\}
$$

и $h_{b, \sigma}^{\bar{\sigma}} \in R$.

Говорят, что вероятностное распределение $\mu^{(n)}$ согласованно, если

$$
\sum_{\sigma^{(n)}} \mu^{(n)}\left(\sigma_{n-1}, \sigma^{(n)}\right)=\mu^{(n-1)}\left(\sigma_{n-1}\right)
$$

для всех $n \geqslant 1$ и $\sigma_{n-1} \in \Omega_{V_{n-1}}$. 
В этом случае существует единственная мера $\mu$ на $\Omega_{V}$ такая, что

$$
\mu\left(\left\{\left.\sigma\right|_{V_{n}}=\sigma_{n}\right\}\right)=\mu^{(n)}\left(\sigma_{n}\right)
$$

для всех $n \geqslant 1$ и $\sigma_{n} \in \Omega_{V_{n}}$.

Рассмотрим конфигурации $\sigma_{0}=\{+,+,+,+\}, \sigma_{1}=\{+,-,+,+\}, \sigma_{2}=\{+,+,-,-\}$, $\sigma_{3}=\{+,-,-,-\},-\sigma_{0}=\{-,-,-,-\},-\sigma_{1}=\{-,+,-,-\},-\sigma_{2}=\{-,-,+,+\}$, $-\sigma_{3}=\{-,+,+,+\}$ на единичном шаре.

Обозначим

$$
\begin{array}{rlrl}
h_{b, \sigma_{0}}^{\sigma_{0}} & =h_{b, 0}, \quad h_{b, \sigma_{1}}^{\sigma_{0}}=h_{b, 1}, & h_{b, \sigma_{2}}^{\sigma_{0}}=h_{b, 2}, & h_{b,-\sigma_{1}}^{\sigma_{1}}=h_{b, 3}, \\
h_{b,-\sigma_{2}}^{\sigma_{1}} & =h_{b, 4}, \quad h_{b,-\sigma_{3}}^{\sigma_{1}}=h_{b, 5}, \quad h_{b, \sigma_{1}}^{-\sigma_{1}}=h_{b, 6}, & h_{b, \sigma_{2}}^{-\sigma_{1}}=h_{b, 7}, \\
h_{b, \sigma_{3}}^{-\sigma_{1}} & =h_{b, 8}, \quad h_{b,-\sigma_{0}}^{-\sigma_{0}}=h_{b, 9}, \quad h_{b,-\sigma_{1}}^{-\sigma_{0}}=h_{b, 10}, & h_{b,-\sigma_{2}}^{-\sigma_{0}}=h_{b, 11} .
\end{array}
$$

Для $a \in M$ через $b$ и $c$ обозначим "прямые потомки" шара $a$ [10]. Следующая теорема дает необходимые и достаточные условия для $h_{b, i}$, при которых выполняется равенство (2.2).

Теорема 1. Пусть $k=2$. Вероятностное распределение $\mu^{(n)}\left(\sigma_{n}\right), n=1,2, \ldots$, в (2.1) является согласованным тогда и только тогда, когда для любого а $\in M$ выполняются следующие равенства:

$$
\begin{aligned}
& y_{a, 0}=y_{a, 6}=\frac{\lambda y_{b, 0}+y_{b, 1}+y_{b, 2}}{y_{b, 0}+y_{b, 1}+y_{b, 2}} \frac{\lambda y_{c, 0}+y_{c, 1}+y_{c, 2}}{y_{c, 0}+y_{c, 1}+y_{c, 2}}, \\
& y_{a, 1}=y_{a, 7}=\frac{\lambda y_{b, 0}+y_{b, 1}+y_{b, 2}}{y_{b, 0}+y_{b, 1}+y_{b, 2}} \frac{y_{c, 3}+y_{c, 4}+1}{y_{c, 0}+y_{c, 1}+y_{c, 2}}, \\
& y_{a, 2}=y_{a, 8}=\frac{y_{b, 3}+y_{b, 4}+1}{y_{b, 0}+y_{b, 1}+y_{b, 2}} \frac{y_{c, 3}+y_{c, 4}+1}{y_{c, 0}+y_{c, 1}+y_{c, 2}}, \\
& y_{a, 3}=y_{a, 9}=\frac{\lambda y_{b, 3}+y_{b, 4}+1}{y_{b, 0}+y_{b, 1}+y_{b, 2}} \frac{\lambda y_{c, 3}+y_{c, 4}+1}{y_{c, 0}+y_{c, 1}+y_{c, 2}}, \\
& y_{a, 4}=y_{a, 10}=\frac{\lambda y_{c, 3}+y_{c, 4}+1}{y_{c, 0}+y_{c, 1}+y_{c, 2}}, \quad y_{a, 5}=1,
\end{aligned}
$$

где $\lambda=e^{J \beta}, \beta=1 / T, y_{a, i}=e^{h_{a, i}-h_{a, 11}}, i=\overline{0,10}$.

ДокАзАтЕльство. Пусть $x$ и $y$ - соседние вершины и на шаре $b(x)$ задана конфигурация $\sigma_{b(x)} \in\left\{ \pm \sigma_{0}, \pm \sigma_{1}, \pm \sigma_{2}, \pm \sigma_{3}\right\}$. Тогда конфигурация $\sigma_{b(y)}$ зависит от $\sigma_{b(x)}$. В таблице заданы все возможные значения $\sigma_{b(y)}$ при фиксированной $\sigma_{b(x)}$.

Заметим, что модель (1.2) теперь может быть рассмотрена как НС-модель со значениями "спина", выбранными из этой таблицы допустимых значений спина на соседних вершинах дерева Кэли.

Обозначим $\bar{M}_{n}=\{b \in M: d(b, \bar{e}) \leqslant n\}, \bar{W}_{n}=\{b \in M: d(b, \bar{e})=n\}$, где $\bar{e}-$ шар радиуса 1 с центром $e$, где $e$ - единичный элемент группы $G_{b}$; тогда $d\left(b_{1}, b_{2}\right)=$ $d\left(c_{b_{1}}, c_{b_{2}}\right)$, где $c_{b}$ обозначает центр шара $b$.

Пусть $\sigma_{n}=\left\{\sigma_{b}, b \in \bar{M}_{n}\right\}, \sigma^{(n)}=\left\{\sigma_{b}, b \in \bar{W}_{n}\right\}$.

В этом случае гамильтониан имеет вид

$$
H\left(\sigma_{n}\right)=-J \sum_{b \in \bar{V}_{n}} U\left(\sigma_{b}\right)=H\left(\sigma_{n-1}\right)-J \sum_{b \in \bar{W}_{n}} U\left(\sigma_{b}\right) .
$$




\begin{tabular}{|c|c|c|}
\hline$\sigma_{b(x)}$ & $\sigma_{b(y)}, \sigma(y)=+1$ & $\sigma_{b(y)}, \sigma(y)=-1$ \\
\hline$\sigma_{0}$ & $\sigma_{0}, \sigma_{1}, \sigma_{2}$ & $\varnothing$ \\
\hline$\sigma_{1}$ & $\sigma_{0}, \sigma_{1}, \sigma_{2}$ & $-\sigma_{1},-\sigma_{2},-\sigma_{3}$ \\
\hline$\sigma_{2}$ & $\sigma_{0}, \sigma_{1}, \sigma_{2}$ & $-\sigma_{1},-\sigma_{2},-\sigma_{3}$ \\
\hline$\sigma_{3}$ & $\varnothing$ & $-\sigma_{1},-\sigma_{2},-\sigma_{3}$ \\
\hline$-\sigma_{0}$ & $\varnothing$ & $-\sigma_{0},-\sigma_{1},-\sigma_{2}$ \\
\hline$-\sigma_{1}$ & $\sigma_{1}, \sigma_{2}, \sigma_{3}$ & $-\sigma_{0},-\sigma_{1},-\sigma_{2}$ \\
\hline$-\sigma_{2}$ & $\sigma_{1}, \sigma_{2}, \sigma_{3}$ & $-\sigma_{0},-\sigma_{1},-\sigma_{2}$ \\
\hline$-\sigma_{3}$ & $\sigma_{1}, \sigma_{2}, \sigma_{3}$ & $\varnothing$ \\
\hline
\end{tabular}

Докажем сначала необходимость сформулированных в теореме условий. Из формулы (2.2) получим

$$
\begin{aligned}
\sum_{\sigma^{(n)}} \mu_{n}\left(\sigma_{n-1}, \sigma^{(n)}\right) & =Z_{n}^{-1} \sum_{\sigma^{(n)}} \exp \left\{\beta H\left(\sigma_{n-1}\right)-J \beta \sum_{b \in \bar{W}_{n}} U\left(\sigma_{b}\right)+\sum_{b \in \bar{W}_{n}} h_{b, \sigma_{b}}^{\sigma_{b}}\right\}= \\
& =Z_{n-1}^{-1} \exp \left\{-\beta H\left(\sigma_{n-1}\right)+\sum_{b \in \bar{W}_{n-1}} h_{a, \sigma_{a}}^{\sigma_{a}}\right\} .
\end{aligned}
$$

Отсюда

$$
L_{n} \sum_{\sigma^{(n)}} \prod_{b \in \bar{W}_{n-1}} \prod_{a \in S(b)} \exp \left\{-J \beta U\left(\sigma_{a}\right)+h_{a, \sigma_{a}}^{\sigma_{a}}\right\}=\prod_{b \in \bar{W}_{n-1}} \exp \left\{h_{b, \sigma_{b}}^{\sigma_{b_{b}}}\right\},
$$

где $L_{n}=Z_{n-1} / Z_{n}$. Следовательно,

$$
L_{n} \prod_{b \in \bar{W}_{n-1}} \prod_{a \in S(b)} \sum_{\sigma_{a}^{(n)}} \exp \left\{-J \beta U\left(\sigma_{a}\right)+h_{a, \sigma_{a}}^{\sigma_{a}}\right\} \mathbf{1}_{\sigma_{n} \in \Omega}=\prod_{b \in \bar{W}_{n-1}} \exp \left\{h_{b, \sigma_{b}}^{\sigma_{b \downarrow}}\right\} .
$$

Отсюда, опираясь на значения, приведенные в таблице, получаем

$$
\begin{aligned}
& e^{h_{a, \sigma_{0}}^{\sigma_{i}}}=L_{n}\left(e^{-\beta U\left(\sigma_{0}\right)+h_{b, \sigma_{0}}^{\sigma_{0}}}+e^{-\beta U\left(\sigma_{1}\right)+h_{b, \sigma_{1}}^{\sigma_{0}}}+e^{-\beta U\left(\sigma_{2}\right)+h_{b, \sigma_{2}}^{\sigma_{0}}}\right) \times \\
& \times\left(e^{-\beta U\left(\sigma_{0}\right)+h_{c, \sigma_{0}}^{\sigma_{0}}}+e^{-\beta U\left(\sigma_{1}\right)+h_{c, \sigma_{1}}^{\sigma_{0}}}+e^{-\beta U\left(\sigma_{2}\right)+h_{c, \sigma_{2}}^{\sigma_{0}}}\right), \quad i=0,1,2 ; \\
& e^{h_{a, \sigma_{1}}^{\sigma_{i}}}=L_{n}\left(e^{-\beta U\left(\sigma_{0}\right)+h_{b, \sigma_{0}}^{\sigma_{1}}}+e^{-\beta U\left(\sigma_{1}\right)+h_{b, \sigma_{1}}^{\sigma_{1}}}+e^{-\beta U\left(\sigma_{2}\right)+h_{b, \sigma_{2}}^{\sigma_{1}}}\right) \times \\
& \times\left(e^{-\beta U\left(-\sigma_{1}\right)+h_{c,-\sigma_{1}}^{\sigma_{1}}}+e^{-\beta U\left(-\sigma_{2}\right)+h_{c,-\sigma_{2}}^{\sigma_{1}}}+e^{-\beta U\left(-\sigma_{3}\right)+h_{c,-\sigma_{3}}^{\sigma_{1}}}\right), \quad i=0,1,2 ; \\
& e^{h_{a, \sigma_{1}}^{-\sigma_{j}}}=L_{n}\left(e^{-\beta U\left(\sigma_{0}\right)+h_{b, \sigma_{0}}^{\sigma_{1}}}+e^{-\beta U\left(\sigma_{1}\right)+h_{b, \sigma_{1}}^{\sigma_{1}}}+e^{-\beta U\left(\sigma_{2}\right)+h_{b, \sigma_{2}}^{\sigma_{1}}}\right) \times
\end{aligned}
$$

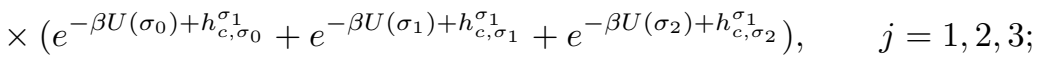

$$
\begin{aligned}
& e^{h_{a, \sigma_{2}}^{\sigma_{i}}}=L_{n}\left(e^{-\beta U\left(-\sigma_{1}\right)+h_{b,-\sigma_{1}}^{\sigma_{2}}}+e^{-\beta U\left(-\sigma_{2}\right)+h_{b,-\sigma_{2}}^{\sigma_{2}}}+e^{-\beta U\left(-\sigma_{3}\right)+h_{b,-\sigma_{3}}^{\sigma_{2}}}\right) \times \\
& \times\left(e^{-\beta U\left(-\sigma_{1}\right)+h_{c,-\sigma_{1}}^{\sigma_{2}}}+e^{-\beta U\left(-\sigma_{2}\right)+h_{c,-\sigma_{2}}^{\sigma_{2}}}+e^{-\beta U\left(-\sigma_{3}\right)+h_{c,-\sigma_{3}}^{\sigma_{2}}}\right), \quad i=0,1,2 ;
\end{aligned}
$$




$$
\begin{aligned}
& e^{h_{a, \sigma_{2}}^{-\sigma_{j}}}=L_{n}\left(e^{-\beta U\left(\sigma_{0}\right)+h_{b, \sigma_{0}}^{\sigma_{2}}}+e^{-\beta U\left(\sigma_{1}\right)+h_{b, \sigma_{1}}^{\sigma_{2}}}+e^{-\beta U\left(\sigma_{2}\right)+h_{b, \sigma_{2}}^{\sigma_{2}}}\right) \times \\
& \times\left(e^{-\beta U\left(-\sigma_{1}\right)+h_{c,-\sigma_{1}}^{\sigma_{2}}}+e^{-\beta U\left(-\sigma_{2}\right)+h_{c,-\sigma_{2}}^{\sigma_{2}}}+e^{-\beta U\left(-\sigma_{3}\right)+h_{c,-\sigma_{3}}^{\sigma_{2}}}\right), \quad j=1,2,3 ; \\
& e^{h_{a, \sigma_{3}}^{-\sigma_{j}}}=L_{n}\left(e^{-\beta U\left(-\sigma_{1}\right)+h_{b,-\sigma_{1}}^{\sigma_{3}}}+e^{-\beta U\left(-\sigma_{2}\right)+h_{b,-\sigma_{2}}^{\sigma_{3}}}+e^{-\beta U\left(-\sigma_{3}\right)+h_{b,-\sigma_{3}}^{\sigma_{3}}}\right) \times
\end{aligned}
$$

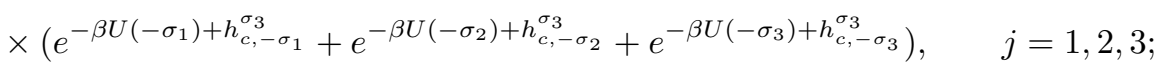

$$
\begin{aligned}
& e^{h_{a,-\sigma_{0}}^{-\sigma_{j}}}=L_{n}\left(e^{-\beta U\left(-\sigma_{0}\right)+h_{b,-\sigma_{0}}^{-\sigma_{0}}}+e^{-\beta U\left(-\sigma_{1}\right)+h_{b,-\sigma_{1}}^{-\sigma_{0}}}+e^{-\beta U\left(-\sigma_{2}\right)+h_{b,-\sigma_{2}}^{-\sigma_{0}}}\right) \times
\end{aligned}
$$

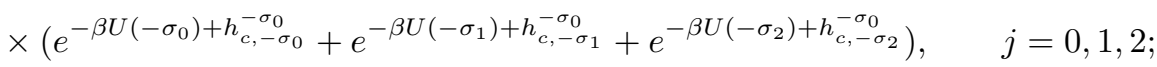

$$
\begin{aligned}
& e^{h_{a,-\sigma_{1}}^{\sigma_{i}}}=L_{n}\left(e^{-\beta U\left(-\sigma_{0}\right)+h_{b,-\sigma_{0}}^{-\sigma_{1}}}+e^{-\beta U\left(-\sigma_{1}\right)+h_{b,-\sigma_{1}}^{-\sigma_{1}}}+e^{-\beta U\left(-\sigma_{2}\right)+h_{b,-\sigma_{2}}^{-\sigma_{1}}}\right) \times \\
& \times\left(e^{-\beta U\left(-\sigma_{0}\right)+h_{c,-\sigma_{0}}^{-\sigma_{1}}}+e^{-\beta U\left(-\sigma_{1}\right)+h_{c,-\sigma_{1}}^{-\sigma_{1}}}+e^{-\beta U\left(-\sigma_{2}\right)+h_{c,-\sigma_{2}}^{-\sigma_{1}}}\right), \quad i=1,2,3 ; \\
& e^{h_{a,-\sigma_{1}}^{-\sigma_{j}}}=L_{n}\left(e^{-\beta U\left(\sigma_{1}\right)+h_{b, \sigma_{1}}^{-\sigma_{1}}}+e^{-\beta U\left(\sigma_{2}\right)+h_{b, \sigma_{2}}^{-\sigma_{1}}}+e^{-\beta U\left(\sigma_{3}\right)+h_{b, \sigma_{3}}^{-\sigma_{1}}}\right) \times \\
& \times\left(e^{-\beta U\left(-\sigma_{0}\right)+h_{c,-\sigma_{0}}^{-\sigma_{1}}}+e^{-\beta U\left(-\sigma_{1}\right)+h_{c,-\sigma_{1}}^{-\sigma_{1}}}+e^{-\beta U\left(-\sigma_{2}\right)+h_{c,-\sigma_{2}}^{-\sigma_{1}}}\right), \quad j=0,1,2 ; \\
& e^{h_{a,-\sigma_{2}}^{\sigma_{i}}}=L_{n}\left(e^{-\beta U\left(\sigma_{1}\right)+h_{b, \sigma_{1}}^{-\sigma_{2}}}+e^{-\beta U\left(\sigma_{2}\right)+h_{b, \sigma_{2}}^{-\sigma_{2}}}+e^{-\beta U\left(\sigma_{3}\right)+h_{b, \sigma_{3}}^{-\sigma_{2}}}\right) \times \\
& \times\left(e^{-\beta U\left(-\sigma_{0}\right)+h_{c,-\sigma_{0}}^{-\sigma_{2}}}+e^{-\beta U\left(-\sigma_{1}\right)+h_{c,-\sigma_{1}}^{-\sigma_{2}}}+e^{-\beta U\left(-\sigma_{2}\right)+h_{c,-\sigma_{2}}^{-\sigma_{2}}}\right), \quad i=1,2,3 ; \\
& e^{h_{a,-\sigma_{2}}^{-\sigma_{j}}}=L_{n}\left(e^{-\beta U\left(\sigma_{1}\right)+h_{b, \sigma_{1}}^{-\sigma_{2}}}+e^{-\beta U\left(\sigma_{2}\right)+h_{b, \sigma_{2}}^{-\sigma_{2}}}+e^{-\beta U\left(\sigma_{3}\right)+h_{b, \sigma_{3}}^{-\sigma_{2}}}\right) \times \\
& \times\left(e^{-\beta U\left(\sigma_{1}\right)+h_{c, \sigma_{1}}^{-\sigma_{2}}}+e^{-\beta U\left(\sigma_{2}\right)+h_{c, \sigma_{2}}^{-\sigma_{2}}}+e^{-\beta U\left(\sigma_{3}\right)+h_{c, \sigma_{3}}^{-\sigma_{2}}}\right), \quad j=0,1,2 ; \\
& e^{h_{a,-\sigma_{3}}^{\sigma_{i}}}=L_{n}\left(e^{-\beta U\left(\sigma_{1}\right)+h_{b, \sigma_{1}}^{-\sigma_{3}}}+e^{-\beta U\left(\sigma_{2}\right)+h_{b, \sigma_{2}}^{-\sigma_{3}}}+e^{-\beta U\left(\sigma_{3}\right)+h_{b, \sigma_{3}}^{-\sigma_{3}}}\right) \times \\
& \times\left(e^{-\beta U\left(\sigma_{1}\right)+h_{c, \sigma_{1}}^{-\sigma_{3}}}+e^{-\beta U\left(\sigma_{2}\right)+h_{c, \sigma_{2}}^{-\sigma_{3}}}+e^{-\beta U\left(\sigma_{3}\right)+h_{c, \sigma_{3}}^{-\sigma_{3}}}\right), \quad i=1,2,3 .
\end{aligned}
$$

Обозначим $z_{a, i}=e^{h_{a, i}}$. Тогда в силу формул $(2.3)$ имеем

$$
\begin{aligned}
z_{a, 0} & =\lambda^{4} L_{n}\left(\lambda z_{b, 0}+z_{b, 1}+z_{b, 2}\right)\left(\lambda z_{c, 0}+z_{c, 1}+z_{c, 2}\right) \\
z_{a, 1} & =\lambda^{4} L_{n}\left(\lambda z_{b, 0}+z_{b, 1}+z_{b, 2}\right)\left(z_{c, 3}+z_{c, 4}+z_{c, 5}\right) \\
z_{a, 2} & =\lambda^{4} L_{n}\left(z_{b, 3}+z_{b, 4}+z_{b, 5}\right)\left(z_{c, 3}+z_{c, 4}+z_{c, 5}\right) \\
z_{a, 3} & =\lambda^{4} L_{n}\left(\lambda z_{b, 9}+z_{b, 10}+z_{b, 11}\right)\left(\lambda z_{c, 9}+z_{c, 10}+z_{c, 11}\right), \\
z_{a, 4} & =\lambda^{4} L_{n}\left(z_{b, 6}+z_{b, 7}+z_{b, 8}\right)\left(\lambda z_{c, 9}+z_{c, 10}+z_{c, 11}\right) \\
z_{a, 5} & =\lambda^{4} L_{n}\left(z_{b, 6}+z_{b, 7}+z_{b, 8}\right)\left(z_{c, 6}+z_{c, 7}+z_{c, 8}\right) \\
z_{a, 6} & =\lambda^{4} L_{n}\left(\lambda z_{b, 0}+z_{b, 1}+z_{b, 2}\right)\left(\lambda z_{c, 0}+z_{c, 1}+z_{c, 2}\right) \\
z_{a, 7} & =\lambda^{4} L_{n}\left(\lambda z_{b, 0}+z_{b, 1}+z_{b, 2}\right)\left(z_{c, 3}+z_{c, 4}+z_{c, 5}\right) \\
z_{a, 8} & =\lambda^{4} L_{n}\left(z_{b, 3}+z_{b, 4}+z_{b, 5}\right)\left(z_{c, 3}+z_{c, 4}+z_{c, 5}\right) \\
z_{a, 9} & =\lambda^{4} L_{n}\left(\lambda z_{b, 9}+z_{b, 10}+z_{b, 11}\right)\left(\lambda z_{c, 9}+z_{c, 10}+z_{c, 11}\right) \\
z_{a, 10} & =\lambda^{4} L_{n}\left(z_{b, 6}+z_{b, 7}+z_{b, 8}\right)\left(\lambda z_{c, 9}+z_{c, 10}+z_{c, 11}\right) \\
z_{a, 11} & =\lambda^{4} L_{n}\left(z_{b, 6}+z_{b, 7}+z_{b, 8}\right)\left(z_{c, 6}+z_{c, 7}+z_{c, 8}\right) .
\end{aligned}
$$

Положим $y_{a, i}=z_{a, i} / z_{a, 11}, i=0,1, \ldots, 10$, и получим

$$
y_{a, 0}=\frac{\lambda y_{b, 0}+y_{b, 1}+y_{b, 2}}{y_{b, 6}+y_{b, 7}+y_{b, 8}} \frac{\lambda y_{c, 0}+y_{c, 1}+y_{c, 2}}{y_{c, 6}+y_{c, 7}+y_{c, 8}},
$$




$$
\begin{aligned}
y_{a, 1} & =\frac{\lambda y_{b, 0}+y_{b, 1}+y_{b, 2}}{y_{b, 6}+y_{b, 7}+y_{b, 8}} \frac{y_{c, 3}+y_{c, 4}+y_{c, 5}}{y_{c, 6}+y_{c, 7}+y_{c, 8}}, \\
y_{a, 2} & =\frac{y_{b, 3}+y_{b, 4}+y_{b, 5}}{y_{b, 6}+y_{b, 7}+y_{b, 8}} \frac{y_{c, 3}+y_{c, 4}+y_{c, 5}}{y_{c, 6}+y_{c, 7}+y_{c, 8}} \\
y_{a, 3} & =\frac{\lambda y_{b, 9}+y_{b, 10}+1}{y_{b, 6}+y_{b, 7}+y_{b, 8}} \frac{\lambda y_{c, 9}+y_{c, 10}+1}{y_{c, 6}+y_{c, 7}+y_{c, 8}}, \\
y_{a, 4}= & \frac{\lambda y_{c, 9}+y_{c, 10}+1}{y_{c, 6}+y_{c, 7}+y_{c, 8}}, \\
y_{a, 5}= & 1, \\
y_{a, 6}= & \frac{\lambda y_{b, 0}+y_{b, 1}+y_{b, 2}}{y_{b, 6}+y_{b, 7}+y_{b, 8}} \frac{\lambda y_{c, 0}+y_{c, 1}+y_{c, 2}}{y_{c, 6}+y_{c, 7}+y_{c, 8}}, \\
y_{a, 7}= & \frac{\lambda y_{b, 0}+y_{b, 1}+y_{b, 2}}{y_{b, 6}+y_{b, 7}+y_{b, 8}} \frac{y_{c, 3}+y_{c, 4}+y_{c, 5}}{y_{c, 6}+y_{c, 7}+y_{c, 8}}, \\
y_{a, 8}= & \frac{y_{b, 3}+y_{b, 4}+y_{b, 5}}{y_{b, 6}+y_{b, 7}+y_{b, 8}} \frac{y_{c, 3}+y_{c, 4}+y_{c, 5}}{y_{c, 6}+y_{c, 7}+y_{c, 8}}, \\
y_{a, 9}= & \frac{\lambda y_{b, 9}+y_{b, 10}+1}{y_{b, 6}+y_{b, 7}+y_{b, 8}} \frac{\lambda y_{c, 9}+y_{c, 10}+1}{y_{c, 6}+y_{c, 7}+y_{c, 8}}, \\
y_{a, 10}= & \frac{\lambda y_{c, 9}+y_{c, 10}+1}{y_{c, 6}+y_{c, 7}+y_{c, 8}} .
\end{aligned}
$$

Заметим, что

$y_{a, 0}=y_{a, 6}, \quad y_{a, 1}=y_{a, 7}, \quad y_{a, 2}=y_{a, 8}, \quad y_{a, 3}=y_{a, 9}, \quad y_{a, 4}=y_{a, 10}, \quad y_{a, 5}=1$.

Следовательно,

$$
\begin{aligned}
y_{a, 0} & =\frac{\lambda y_{b, 0}+y_{b, 1}+y_{b, 2}}{y_{b, 0}+y_{b, 1}+y_{b, 2}} \frac{\lambda y_{c, 0}+y_{c, 1}+y_{c, 2}}{y_{c, 0}+y_{c, 1}+y_{c, 2}}, \\
y_{a, 1} & =\frac{\lambda y_{b, 0}+y_{b, 1}+y_{b, 2}}{y_{b, 0}+y_{b, 1}+y_{b, 2}} \frac{y_{c, 3}+y_{c, 4}+1}{y_{c, 0}+y_{c, 1}+y_{c, 2}}, \\
y_{a, 2} & =\frac{y_{b, 3}+y_{b, 4}+1}{y_{b, 0}+y_{b, 1}+y_{b, 2}} \frac{y_{c, 3}+y_{c, 4}+1}{y_{c, 0}+y_{c, 1}+y_{c, 2}}, \\
y_{a, 3} & =\frac{\lambda y_{b, 3}+y_{b, 4}+1}{y_{b, 0}+y_{b, 1}+y_{b, 2}} \frac{\lambda y_{c, 3}+y_{c, 4}+1}{y_{c, 0}+y_{c, 1}+y_{c, 2}}, \\
y_{a, 4} & =\frac{\lambda y_{c, 3}+y_{c, 4}+1}{y_{c, 0}+y_{c, 1}+y_{c, 2}} .
\end{aligned}
$$

Для доказательства достаточности получим из системы (2.9) при учете (2.8) формулы (2.5)-(2.7), т.е. (2.2). Теорема доказана.

\section{3. ТРАНСЛЯЦИОННО-ИНВАРИАНТНЫЕ МЕРЫ ГИББСА}

В этом разделе мы рассмотрим трансляционно-инвариантные (ТИ) меры для модели (1.2) на дереве Кэли. Заметим, что ТИ-меры Гиббса соответствуют решениям (2.4) с $y_{a, i}=y_{i}$ при всех $a \in M$ и $i=0,1,2,3,4$. В формулах (2.9) положим 
$y_{a, i}=y_{i} \in R_{+}, a \in M$, и получим

$$
\begin{aligned}
& y_{0}=\left(\frac{\lambda y_{0}+y_{1}+y_{2}}{y_{0}+y_{1}+y_{2}}\right)^{2}, \\
& y_{1}=\left(\frac{\lambda y_{0}+y_{1}+y_{2}}{y_{0}+y_{1}+y_{2}}\right)\left(\frac{y_{3}+y_{4}+1}{y_{0}+y_{1}+y_{2}}\right), \\
& y_{2}=\left(\frac{y_{3}+y_{4}+1}{y_{0}+y_{1}+y_{2}}\right)^{2}, \\
& y_{3}=\left(\frac{\lambda y_{3}+y_{4}+1}{y_{0}+y_{1}+y_{2}}\right)^{2}, \\
& y_{4}=\frac{\lambda y_{3}+y_{4}+1}{y_{0}+y_{1}+y_{2}},
\end{aligned}
$$

где $\lambda>0$.

ТЕОрема 2. Для модели (1.2) существует $\lambda_{\mathrm{cr}}(\approx 1.8055)$ такое, что при (11$\sqrt{21}) / 8<\lambda<\lambda_{\mathrm{cr}}$ существует не менее одной ТИ-меры Гиббса, при $\lambda=\lambda_{\mathrm{cr}}-$ не менее двух, а при $\lambda_{\mathrm{cr}}<\lambda<(11+\sqrt{21}) / 8$ - не менее трех ТИ-мер Гиббса.

ДоказАТЕльство. Из системы (3.1) после несложных вычислений получаем

$$
\begin{aligned}
y_{4}=y= & \left\{\left(\lambda y^{2}+y+1\right)^{2}\left[\lambda y^{2}+y+1 \pm \sqrt{D}\right]^{2}+\right. \\
& +2(\lambda-1) y^{2}\left(y^{2}+y+1\right)\left(\lambda y^{2}+y+1\right)\left[\lambda y^{2}+y+1 \pm \sqrt{D}\right]+ \\
& \left.+4(\lambda-1)^{2} y^{4}\left(y^{2}+y+1\right)^{2}\right\} /\left\{4(\lambda-1)^{2}\left(\lambda y^{2}+y+1\right)^{3}\right\}=\varphi_{ \pm}(y, \lambda),
\end{aligned}
$$

где $D=\left(\lambda y^{2}+y+1\right)\left(\lambda y^{2}+(5-4 \lambda) y+1\right)$.

Заметим, что из условия $D \geqslant 0$ следует $(11-\sqrt{21}) / 8 \leqslant \lambda \leqslant(11+\sqrt{21}) / 8$. Легко видеть, что $\varphi_{-}(1, \lambda)<0$ и $\varphi_{-}(+\infty, \lambda)=+\infty$ для любого $\lambda>0$. Следовательно, $\varphi_{-}(y, \lambda)=y$ имеет не менее одного решения для любого $\lambda \geqslant(11-\sqrt{21}) / 8 \geqslant 0$. При $\lambda=1.86$ видно, что $\varphi_{+}(0, \lambda)>0, \varphi_{+}(1, \lambda)<0, \varphi_{+}(+\infty, \lambda)=+\infty$, т. е. уравнение $y=\varphi_{+}(y)$ имеет не менее двух решений (на рисунке показан график функции $\varphi_{+}(y)$ при различных значениях $\lambda$ ). Приблизительное значение $\lambda_{\mathrm{cr}} \approx 1.8055$ получено с помощью компьютера.

Теорема доказана.

Применяя методы, предложенные в работах [4],[6], легко доказать следующую теорему.

Теорема 3. При $\lambda_{\mathrm{cr}} \leqslant \lambda<(11+\sqrt{21}) / 8$ существует континуум нетрансляиионно-инвариантных мер Гиббса.

Предположим, что

$$
y_{a, 0}=y_{a, 3}, \quad y_{a, 1}=y_{a, 4}, \quad y_{a, 2}=1, \quad a \in M .
$$

Тогда из системы (2.7) получаем

$$
\begin{aligned}
y_{a, 0} & =\frac{\lambda y_{b, 0}+y_{b, 1}+1}{y_{b, 0}+y_{b, 1}+1} \frac{\lambda y_{c, 0}+y_{c, 1}+1}{y_{c, 0}+y_{c, 1}+1}, \\
y_{a, 1} & =\frac{\lambda y_{b, 0}+y_{b, 1}+1}{y_{b, 0}+y_{b, 1}+1} .
\end{aligned}
$$



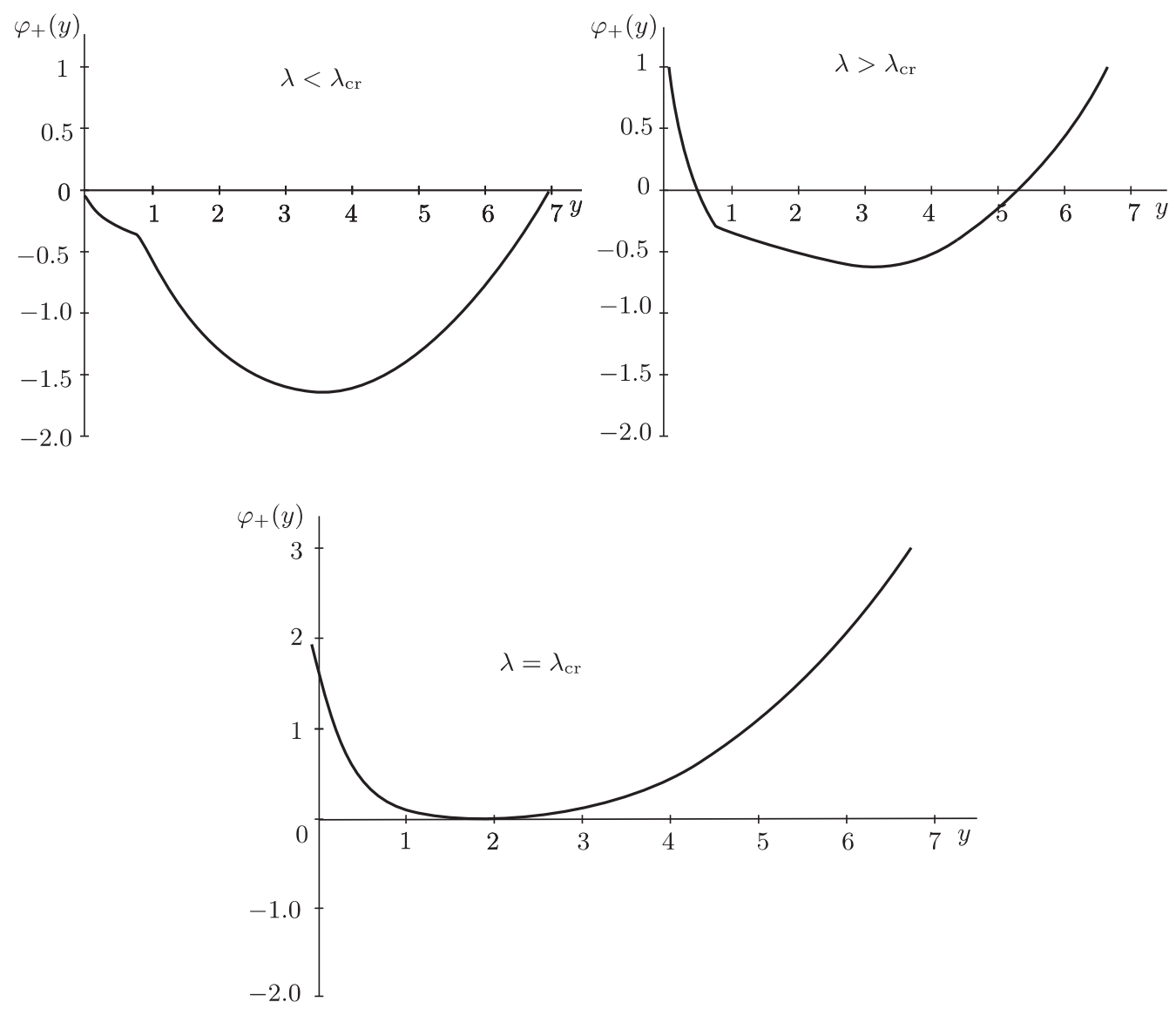

Докажем следующие леммы.

Лемма 1. 1. Пусть $\lambda>1$. Если $y_{a}=\left(y_{a, 0}, y_{a, 1}\right), a \in M$, является решением системы (3.3), то $y_{i}^{-} \leqslant y_{a, i} \leqslant y_{i}^{+}$для любого $i=0,1, a \in M$, где $\left(y_{0}^{-}, y_{0}^{+}, y_{1}^{-}, y_{1}^{+}\right)-$ решение системы

$$
\begin{aligned}
& y_{0}^{-}=\left(\frac{\lambda y_{0}^{-}+y_{1}^{+}+1}{y_{0}^{-}+y_{1}^{+}+1}\right)^{2}, \\
& y_{0}^{+}=\left(\frac{\lambda y_{0}^{+}+y_{1}^{-}+1}{y_{0}^{+}+y_{1}^{-}+1}\right)^{2}, \\
& y_{1}^{-}=\frac{\lambda y_{0}^{-}+y_{1}^{+}+1}{y_{0}^{-}+y_{1}^{+}+1}, \\
& y_{1}^{+}=\frac{\lambda y_{0}^{+}+y_{1}^{-}+1}{y_{0}^{+}+y_{1}^{-}+1} .
\end{aligned}
$$

2. Пусть $\lambda<1$. Если $y_{a}=\left(y_{a, 0}, y_{a, 1}\right), a \in M$, является решением системы (3.3), то $y_{i}^{-} \leqslant y_{a, i} \leqslant y_{i}^{+}$для любого $i=0,1, a \in M$, где $\left(y_{0}^{-}, y_{0}^{+}, y_{1}^{-}, y_{1}^{+}\right)$- решение 
системь

$$
\begin{aligned}
& y_{0}^{-}=\left(\frac{\lambda y_{0}^{+}+y_{1}^{-}+1}{y_{0}^{+}+y_{1}^{-}+1}\right)^{2}, \\
& y_{0}^{+}=\left(\frac{\lambda y_{0}^{-}+y_{1}^{+}+1}{y_{0}^{-}+y_{1}^{+}+1}\right)^{2}, \\
& y_{1}^{-}=\frac{\lambda y_{0}^{+}+y_{1}^{-}+1}{y_{0}^{+}+y_{1}^{-}+1}, \\
& y_{1}^{+}=\frac{\lambda y_{0}^{-}+y_{1}^{+}+1}{y_{0}^{-}+y_{1}^{+}+1} .
\end{aligned}
$$

ДокАзАтельство. Докажем п. 1 леммы. Очевидно, что $1<y_{a, 0}<\lambda^{2}, 1<y_{a, 1}<$ $\lambda$ для любого $a \in M$.

Рассмотрим функцию

$$
f(u, v)=\frac{\lambda u+v+1}{u+v+1},
$$

где $1<u<\lambda^{2}, 1<v<\lambda$. Нетрудно видеть, что $(2 \lambda+1) /(\lambda+2)<f(u, v)<\lambda$. Таким образом, для $y_{a, i}$ мы получаем $((2 \lambda+1) /(\lambda+2))^{2}<y_{a, 0}<\lambda^{2},(2 \lambda+1) /(\lambda+2)<$ $y_{a, 1}<\lambda$. Теперь рассмотрим $f(u, v)$ с условиями $((2 \lambda+1) /(\lambda+2))^{2}<u<\lambda^{2}$, $(2 \lambda+1) /(\lambda+2)<v<\lambda$. Итерируя, получаем $y_{i, n}^{-} \leqslant y_{a, i} \leqslant y_{i, n}^{+}, i=0,1$. Здесь $y_{i, n}^{ \pm}$, $i=0,1, n=1,2, \ldots$, удовлетворяют равенствам

$$
\begin{aligned}
& y_{0, n+1}^{-}=\left(\frac{\lambda y_{0, n}^{-}+y_{1, n}^{+}+1}{y_{0, n}^{-}+y_{1, n}^{+}+1}\right)^{2}, \\
& y_{0, n+1}^{+}=\left(\frac{\lambda y_{0, n}^{+}+y_{1, n}^{-}+1}{y_{0, n}^{+}+y_{1, n}^{-}+1}\right)^{2}, \\
& y_{1, n+1}^{-}=\frac{\lambda y_{0, n}^{-}+y_{1, n}^{+}+1}{y_{0, n}^{-}+y_{1, n}^{+}+1}, \\
& y_{1, n+1}^{+}=\frac{\lambda y_{0, n}^{+}+y_{1, n}^{-}+1}{y_{0, n}^{+}+y_{1, n}^{-}+1},
\end{aligned}
$$

где $y_{0,1}^{-}=1, y_{0,1}^{+}=\lambda^{2}, y_{1,1}^{-}=1, y_{1,1}^{+}=\lambda$. Видно, что $y_{i, n}^{-}, i=0,1\left(y_{i, n}^{+}, i=\right.$ $0,1)$, является возрастающей (убывающей) и ограниченной последовательностью. Поэтому существует $\lim _{n \rightarrow \infty} y_{i, n}^{ \pm}=y_{i}^{ \pm} i=0,1$. Первый пункт леммы доказан.

Аналогично доказывается второй пункт леммы.

Лемма 2. Если $y=\left(y_{0}^{-}, y_{0}^{+}, y_{1}^{-}, y_{1}^{+}\right)$- решение системъ $(3.4)$, то $y_{0}^{-}=y_{0}^{+}$тогда и только тогда, когда $y_{1}^{-}=y_{1}^{+}$.

ДокАЗАтЕЛьСтво. Из системы (3.4) имеем

$$
\begin{array}{r}
\left(y_{0}^{-}-y_{0}^{+}\right)\left(A B-1+A B y_{1}^{+}\right)+A B y_{0}^{-}\left(y_{1}^{-}-y_{1}^{+}\right)=0, \\
\left(y_{0}^{-}-y_{0}^{+}\right)\left(1+y_{1}^{+}\right) B+\left(B y_{0}^{-}-1\right)\left(y_{1}^{-}-y_{1}^{+}\right)=0,
\end{array}
$$

где

$$
A=\frac{\lambda y_{0}^{-}+y_{1}^{+}+1}{y_{0}^{-}+y_{1}^{+}+1}+\frac{\lambda y_{0}^{+}+y_{1}^{-}+1}{y_{0}^{+}+y_{1}^{-}+1}>0, \quad B=\frac{\lambda-1}{\left(y_{0}^{-}+y_{1}^{+}+1\right)\left(y_{0}^{+}+y_{1}^{-}+1\right)}>0 .
$$


Если $y_{0}^{-}=y_{0}^{+}$, то из первого уравнения системы (3.5) имеем $y_{1}^{-}=y_{1}^{+}$. Если $y_{1}^{-}=y_{1}^{+}$, то из второго уравнения системы (3.5) имеем $y_{0}^{-}=y_{0}^{+}$. Лемма доказана.

\section{4. ПЕРИОДИЧЕСКИЕ МЕРЫ ГИББСА}

В настоящем разделе мы рассматриваем периодические меры Гиббса в случае выполнения условия (3.2). Запишем систему (3.3) в следующем виде:

$$
\begin{aligned}
h_{a, 0} & =\ln \left(\frac{\lambda e^{h_{b, 0}}+e^{h_{b, 1}}+1}{e^{h_{b, 0}}+e^{h_{b, 1}}+1} \frac{\lambda e^{h_{c, 0}}+e^{h_{c, 1}}+1}{e^{h_{c, 0}}+e^{h_{c, 1}}+1}\right), \\
h_{a, 1} & =\ln \frac{\lambda e^{h_{b, 0}}+e^{h_{b, 1}}+1}{e^{h_{b, 0}}+e^{h_{b, 1}}+1}
\end{aligned}
$$

где $h_{a, i}=\ln y_{a, i}, i=0,1$, и исследуем ее периодические решения.

ОПРЕДЕЛЕНИЕ 1 . Пусть $H_{0}$ - подгруппа группы $G_{k}$. Совокупность векторов $h=\left\{h_{a}=\left(h_{a, 0}, h_{a, 1}\right): a \in G_{k}\right\}$ называется $H_{0}$-периодической, если $h_{y a, i}=h_{a, i}$ для любых $a \in G_{k}$ и $y \in H_{0}, i=0,1$.

ОПРЕДЕЛЕНИЕ 2. Мера Гиббса, соответствующая $H_{0}$-периодической совокупности векторов, называется $H_{0}$-периодической.

Определим $F: h=\left(h_{0}, h_{1}\right) \rightarrow F(h) \in R$ следующим образом:

$$
F(h)=\ln \frac{\lambda e^{h_{0}}+e^{h_{1}}+1}{e^{h_{0}}+e^{h_{1}}+1} .
$$

Пусть $G_{k}^{(2)}$ - подгруппа группы $G_{k}$, состоящая из слов четной длины. Очевидно, что $G_{k}^{(2)}$ является подгруппой индекса 2 .

Пусть

$$
h_{a, i}=\left\{\begin{array}{lll}
h_{i}, & \text { если } & c_{a} \in G_{2}^{(2)}, \\
h_{i}^{\prime}, & \text { если } & c_{a} \in G_{2} \backslash G_{2}^{(2)},
\end{array} \quad i=0,1 .\right.
$$

Тогда из (4.1) имеем

$$
\begin{aligned}
& h_{0}=2 \ln \frac{\lambda e^{h_{0}^{\prime}}+e^{h_{1}^{\prime}}+1}{e^{h_{0}^{\prime}}+e^{h_{1}^{\prime}}+1}, \\
& h_{0}^{\prime}=2 \ln \frac{\lambda e^{h_{0}}+e^{h_{1}}+1}{e^{h_{0}}+e^{h_{1}}+1}, \\
& h_{1}=\ln \frac{\lambda e^{h_{0}^{\prime}}+e^{h_{1}^{\prime}}+1}{e^{h_{0}^{\prime}}+e^{h_{1}^{\prime}}+1} \\
& h_{1}^{\prime}=\ln \frac{\lambda e^{h_{0}}+e^{h_{1}}+1}{e^{h_{0}}+e^{h_{1}}+1} .
\end{aligned}
$$

Теорема 4. Для модели (1.2) при всех $\lambda>0$ и выполнении условия (3.2) существует единственная $G_{2}^{(2)}$-периодическая мера Гиббса. Более того, эта мера совпадает с ТИ-мерой Гиббса. 
ДоказАтельство. Рассмотрим случай $h_{0}=h_{0}^{\prime}, h_{1}=h_{1}^{\prime}$. Из системы (4.2) получаем

$$
\begin{aligned}
& h_{0}=2 \ln \frac{\lambda e^{h_{0}}+e^{h_{1}}+1}{e^{h_{0}}+e^{h_{1}}+1}, \\
& h_{1}=\ln \frac{\lambda e^{h_{0}}+e^{h_{1}}+1}{e^{h_{0}}+e^{h_{1}}+1} .
\end{aligned}
$$

Эта система уравнений имеет единственное решение $\left(h_{0}^{*}, h_{1}^{*}\right)$. Действительно, из (4.3) следует $e^{3 h_{1}}+(1-\lambda) e^{2 h_{1}}-1=0$. Пусть $e^{h_{1}}=z$, тогда $Q(z)=z^{3}+(1-\lambda) z^{2}-1=0$.

Известно, что число положительных решений уравнения $z^{3}+(1-\lambda) z^{2}-1=0$ не превосходит число перемен знаков коэффициентов, т.е. $1,1-\lambda,-1$ (см. монографию [11], с. 28). Следовательно, $Q(z)=0$ имеет единственное решение.

Теперь рассмотрим случай $h_{0} \neq h_{0}^{\prime}, h_{1} \neq h_{1}^{\prime}$. Из системы (4.2) имеем

$$
\begin{aligned}
& h_{1}=\ln \frac{\lambda e^{2 h_{1}^{\prime}}+e^{h_{1}^{\prime}}+1}{e^{2 h_{1}^{\prime}}+e^{h_{1}^{\prime}}+1}, \\
& h_{1}^{\prime}=\ln \frac{\lambda e^{2 h_{1}}+e^{h_{1}}+1}{e^{2 h_{1}}+e^{h_{1}}+1},
\end{aligned}
$$

откуда при $z=e^{h_{1}}$ получаем многочлен

$$
\begin{aligned}
P(z)= & \left(\lambda^{2}+\lambda+1\right) z^{5}-\left(\lambda^{3}-2 \lambda-2\right) z^{4}-\left(2 \lambda^{2}-2 \lambda-3\right) z^{3}- \\
& -\left(2 \lambda^{2}+2 \lambda-1\right) z^{2}-(2 \lambda+1) z-\lambda-2=0,
\end{aligned}
$$

который легко разложить: $P(z)=Q(z) M(z)$, где $M(z)=\left(\lambda^{2}+\lambda+1\right) z^{2}+(2 \lambda+1) z+$ $\lambda+2$.

Так как $Q(z)=0$ имеет единственное решение и $M(z)=0$ не имеет решения, то $P(z)=0$ имеет единственное решение. Теорема доказана.

Число букв $a_{i}, i=\overline{1, k+1}$, участвующих в несократимой записи слова $x$, обозначим через $\omega_{x}\left(a_{i}\right)$.

Пусть $\varnothing=A \subseteq N_{2}=\{1,2,3\}$ и $H_{A}=\left\{x \in G_{2}: \sum_{i \in A} \omega_{x}\left(a_{i}\right)\right.$ - четное число $\}$. Очевидно, что $H_{A}$ является подгруппой индекса 2 [1].

Пусть

$$
h_{a, j}=\left\{\begin{array}{ll}
h_{j}, & c_{a} \in H_{A}, \\
h_{j}^{\prime}, & c_{a} \in G_{2} \backslash H_{A},
\end{array} \quad j=0,1 .\right.
$$

Заметим, что

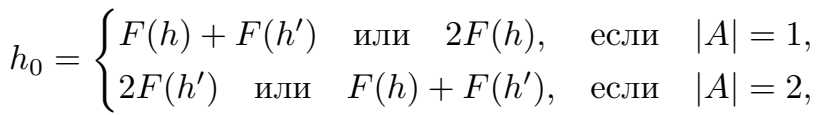

$$
\begin{aligned}
& h_{0}^{\prime}=\left\{\begin{array}{lccc}
F(h)+F\left(h^{\prime}\right) & \text { или } 2 F\left(h^{\prime}\right), & \text { если } & |A|=1, \\
2 F(h) \text { или } & F(h)+F\left(h^{\prime}\right), & \text { если } & |A|=2,
\end{array}\right. \\
& h_{1}=F(h) \quad \text { или } F\left(h^{\prime}\right) \text {, } \\
& h_{1}^{\prime}=F\left(h^{\prime}\right) \quad \text { или } \quad F(h) \text {. }
\end{aligned}
$$

Случай $|A|=3$ соответствует случаю $H_{A}=G_{2}^{(2)}$, рассмотренному выше. 
Теорема 5. Для модели (1.2) при условии (3.2) существует несчетное число $H_{A}$-периодических мер Гиббса. Более того, эти меры не являются ТИ-мерами и $G_{2}^{(2)}$-периодическими.

ДокАзАТЕЛЬСтво. Из уравнения (4.5) следует, что система имеет решения тогда и только тогда, когда $F(h)=F\left(h^{\prime}\right)$.

Отсюда $t_{0}\left(s_{1}-t_{1}\right)+\left(t_{0}-s_{0}\right)\left(t_{1}+1\right)=0$, где $t_{i}=e^{h_{i}}, s_{i}=e^{h_{i}^{\prime}}, i=0,1$. Обозначим $s_{1}-t_{1}=\alpha$ и получим $s_{1}=t_{1}+\alpha, s_{0}=t_{0}+\alpha t_{0} /\left(t_{1}+1\right)$. Из уравнения (4.5) получаем $t_{0}=\left(1-t_{1}^{2}\right) /\left(t_{1}-\lambda\right)$ и $t_{1}^{3}+(1-\lambda) t_{1}^{2}-1=0$. Это уравнение имеет единственное положительное решение $t_{1}^{*}$. Следовательно, $t_{1}=t_{1}^{*}, t_{0}=t_{0}^{*}=\left(1-\left(t_{1}^{*}\right)^{2}\right) /\left(t_{1}^{*}-\lambda\right)$, $s_{1}=s_{1}^{*}(\alpha)=t_{1}^{*}+\alpha, s_{0}=s_{0}^{*}(\alpha)=t_{0}^{*}+\alpha t_{0}^{*} /\left(t_{1}^{*}+1\right), \alpha \in R$.

Таким образом, для любого $\alpha \geqslant 0$ существует решение $\left(\ln t_{0}^{*}, \ln t_{1}^{*}, \ln s_{0}^{*}(\alpha), \ln s_{1}^{*}(\alpha)\right)$ системы (4.5). Более того, эти решения различны при различных $\alpha$. Теорема доказана.

ЗАмечАниЕ. Заметим, что результат теоремы 5 является новым для модели (1.2), так как для ранее изученных моделей (см., например, работы [1], [3]-[9], [12]) не существовало $H_{A}$-периодических мер Гиббса при $H_{A} \neq G_{k}^{(2)}$. Контурный метод доказательства этого результата приведен в работе [2].

Благодарности. У. А. Розиков благодарит The Abdus Salam International Center for Theoretical Physics (ICTP) (Trieste, Italy) за финансовую поддержку пребывания в ICTP (2010), а также TWAS (TWAS Research Grant 09-009 RG/MATHS/AS_IUNES-CO FR: 3240230333).

\section{Список литературы}

[1] Н. Н. Ганиходжаев, У. А. Розиков, ТМФ, 111:1 (1997), 109-117.

[2] U. A. Rozikov, J. Stat. Phys., 130:4 (2008), 801-813, arXiv: math-ph/0611038.

[3] H.-O. Georgii, Gibbs Measures and Phase Transitions, De Gruyter Stud. Math., 9, Walter de Gruyter, Berlin, 1988.

[4] П. М. Блехер, Н. Н. Ганиходжаев, ТВП, 35:2 (1990), 220-230.

[5] P. M. Bleher, J. Ruiz, V. A. Zagrebnov, J. Stat. Phys., 79:1-2 (1995), 473-482.

[6] У. А. Розиков, ТMФ, 118:1 (1999), 95-104.

[7] М. М. Рахматуллаев, У. А. Розиков, ТМФ, 156:2 (2008), 292-302.

[8] N. N. Ganikhodjaev, U. A. Rozikov, Math. Phys. Anal. Geom., 12:2 (2009), 141-156.

[9] J. B. Martin, U. A. Rozikov, Yu. M. Suhov, J. Nonlin. Math. Phys., 12:3 (2005), 432-448.

[10] U. A. Rozikov, J. Stat. Phys., 122:2 (2006), 217-235.

[11] В. В. Прасолов, Многочленьь, МЦНМО, М., 2003.

[12] U. A. Rozikov, Yu. M. Suhov, Infin. Dimens. Anal. Quantum Probab. Relat. Top., 9:3 (2006), 471-488.

Поступила в редакцию 11.10.2010, после доработки 29.11.2010 